(c) 2005 International Press

Adv. Theor. Math. Phys. 9 (2005) 667-701

\title{
One ring to rule them all and in the darkness bind them?
}

\author{
Iosif Bena ${ }^{1}$ and Nicholas P. Warner ${ }^{2}$ \\ ${ }^{1}$ School of Natural Sciences, Institute for Advanced Study, \\ Princeton, NJ 08540 \\ iosif@sns.ias.edu \\ ${ }^{2}$ Department of Physics and Astronomy, University of Southern California, \\ Los Angeles, CA 90089, USA \\ warner@usc.edu
}

\begin{abstract}
We construct all 11-dimensional, three-charge BPS solutions that preserve a fixed, standard set of supersymmetries. Our solutions include all BPS three-charge rotating black holes, black rings, supertubes, as well as arbitrary superpositions of these objects. We find very large families of black rings and supertubes with profiles that follow arbitrary closed curves in the spatial $\mathbb{R}^{4}$ transverse to the branes. The black rings copiously violate black-hole uniqueness. The supertube solutions are completely regular and generically have small curvature. They also have the same asymptotics as the three-charge black hole; so they might be mapped to microstates of the D1-D5-p system and used to explain the entropy of this black hole.
\end{abstract}

e-print archive: http://lanl.arXiv.org/abs/hep-th/0408106 


\section{Introduction}

One of the more surprising features of supergravity is the existence of large families of supersymmetric solutions that preserve the same class of supersymmetries. Some of these families of solutions arise from D-brane configurations that are assembled so as to create supertubes and black holes. The supersymmetries of these solutions are then determined by the canonical projectors associated with each set of D-branes.

For the D1-D5 system, the smooth bulk supergravity solutions dual to microstates of the boundary CFT have been classified and shown to precisely account for the entropy of this theory [24]. While these results are extremely interesting and suggestive descriptions of black-hole microstates, the D1-D5 system does not give rise to "true" black holes with non-zero horizon area. The most direct way to find whether black-hole microstates are smooth supergravity solutions is to consider the three-charge black hole, which has non-trivial event horizons. For these black holes, we know [29] that the statistical ensemble of the microstates of the boundary theory can exactly account for the entropy. It is therefore fascinating to see if Mathur's picture [27] can be extended to these solutions. If one could find a smooth, threecharge geometry with no horizon that is dual to every microstate of the boundary theory, then our picture of black holes would change drastically. A black hole would be nothing more than a classical effective description of the statistical ensemble of microstate geometries, and puzzles like the information paradox would be easily explained.

A few steps towards extending the "one geometry per microstate" picture to the three-charge case have been made by analyzing these configurations via the Born-Infeld action of the component branes [2], by perturbing or doing spectral flow on two-charge solutions $[17,23,27]$, or by constructing families of three-charge solutions with enhanced symmetry $[1,10]$. So far, all these steps indicate that the "one geometry per microstate" picture of black-hole entropy passes quite a few rather non-trivial consistency checks. However, in order to prove this conjecture, one needs to find and classify all supergravity solutions that have the same supersymmetries and charges as the three-charge black hole and to map these solutions to microstates of the boundary theory.

As one can see from the physics of three-charge supertubes, the most generic three-charge solutions have three dipole charges that do not affect their supersymmetry. Unlike the two-charge supertube solutions $[8,24,25$, 26], these solutions cannot be found by usual solution generating techniques because the three charges can be generically dissolved into fluxes (as in the Klebanov-Strassler solution [21]). Put differently, the three-charge solutions 
seem to be intrinsically non-linear: the Maxwell fields interact non-linearly with one another because such multi-component solutions cannot avoid the non-linearities of the supergravity action.

To find the general three-charge solutions, one therefore needs to use more powerful methods. The first simplification is to work in a "duality frame" that treats all three charges on the same footing. That is, we will work with three sets of M2-branes that intersect only along the time axis. One can then obtain an exact solution of the physically more interesting D1-D5-p system by compactification and T-dualities. The other main ingredient in our search for these solutions is to use directly the fact that they preserve the same supersymmetries as the three sets of M2-branes that give rise to their asymptotic charges. This is essential if the solutions are to represent microstates of the three-charge black hole. We then make heavy use of the requirement that mutually BPS brane probes should feel no force to highly restrict the metric and Maxwell fields of our backgrounds. Having done this, we use the explicit form of their Killing spinors to find linear, first-order differential relations between the metric and the Maxwell fields. As in other involved supergravity solutions [18], knowing the Killing spinors greatly reduces the complexity of the problem. We immediately find that a combination of the Maxwell fields and vielbeins simply has to be self-dual in the $\mathbb{R}^{4}$ transverse to the branes. We then use the equations of motion of the four-form to find a set of modified harmonic equations satisfied by the metric coefficients.

The resulting system of equations is linear, provided one solves it in the right order; at each step, the equations are sourced by quadratic combinations of solutions to previously solved equations. Note that this makes these solutions amenable to "entropy counting:" since the equations are linear, one can hope to easily superpose and combine solutions to create and then count microstates. If the equations were non-linear, then one could not easily do this, and the non-linearities could greatly constrain the solution space. Thus, the conceptual simplicity of the two-charge solution persists with three charges. Each step involves solving linear equations with known sources, and the result then completely solves the M-theory equations of motion while preserving the four supersymmetries of the three underlying sets of M2-branes.

The resulting set of equations reminds one of the equations satisfied by all supersymmetric backgrounds of minimal five-dimensional supergravity [19] and reduce to these equations when all the charges and all the dipole charges are, respectively, equal. ${ }^{1}$

\footnotetext{
${ }^{1}$ After this paper was submitted to the arXiv, we have been informed by Reall [16] that these equations have also been obtained in work that classifies solutions of five-dimensional $U(1)^{3}$ invariant supergravity.
} 
As we will see, our system of equations allows one to construct huge classes of solutions and indicates that one can find a very large number of BPS black rings. Basically, one is completely free to choose the dipole charges to have a ring profile of any shape and orientation in $\mathbb{R}^{4}$ and then distribute fundamental (M2-brane) charges with arbitrary densities along the ring. Having done this, one can find the corresponding solutions by a linear process.

We illustrate this explicitly for the $U(1) \times U(1)$ invariant black ring. The solution is given by specifying three charges, $Q_{j}$, three dipole moments, $q_{j}$, and the radius. The asymptotic charges are the $Q_{j}$ and the two angular momenta (4.35). Given the charges and angular momenta, there are thus two free parameters, one of which determines the area of the horizon. Moreover, for each set of asymptotic charges, there exists a one-parameter family of rings with zero horizon area, which are thus three-charge supertubes. These supertube solutions are regular and the Ricci scalar is bounded by $\left(q_{1} q_{2} q_{3}\right)^{-1 / 3}$. Hence, if one chooses a solution with sufficiently large dipole charges, then the curvature can be kept small enough to remain well within the validity of the supergravity approximation. Our solutions generalize the recent equal-charge BPS black-ring solution [10] and reduce in near ring limit to the flat-ring metric obtained in [1]. They also open up the possibility of extending the recent interesting work on non-BPS black rings [7] to solutions without $U(1) \times U(1)$ invariance.

While we have analyzed the black ring in some detail, we stress that our equations admit solutions with arbitrary profiles. These solutions will generically possess non-trivial horizons. However, as one approaches the profile that sources the dipoles, the solution will look just like the ring solution, and we expect there will be a free parameter that will enable us to set the horizon area to zero, and recover a completely regular solution with no horizon, but with arbitrary profile.

These latter solutions have all the right properties to be the microstate geometries that give rise to the three-charge black hole: they have the same asymptotic charges, they preserve the same supersymmetries, they have small curvature everywhere, and they have no close time-like curves or event horizons. One can moreover map these geometries to chiral null models $[10,15]$ and show they are exact string backgrounds [31]. Of course, as with all BPS configurations, there is subtlety in distinguishing simple superpositions of independent BPS states from the true bound states. One way to achieve this is to match each bulk solution with a microstate of the boundary theory and then verify that the latter really represents a bound state. However, both the two-charge story and the fact that certain supertubes have a consistent Born-Infeld description hint quite strongly that three-charge 
supertubes - solutions with only one ring of dipole charges, all the M2brane charges sourced on the ring, and zero entropy - will be bound states.

If supertube geometries are dual to microstates of the boundary D1D5-p CFT, then how are we to interpret the black rings? If the picture of black-hole hair advanced in [27] is true, then 27 black rings describe ensembles of these supertube microstates. The fact that such a large number of black rings exists strongly supports this interpretation. Indeed, one may try to estimate the entropy of all black rings with fixed charges and angular momenta and see if this overcounts the boundary entropy. If this is true - and the very large families of black rings we find suggest it is - it implies that a given microstate can be contained into a very large number of black rings. The fact that black rings describe sets of nearby microstate geometries is also a very natural explanation of some of the ring solutions that can apparently change from black ring to supertube and back as one moves along the ring.

Aside from the possible duality between the geometries we construct and microstates of the D1-D5-p system, our solutions are also interesting in and of themselves for several reasons. First, it is quite amazing that there exists such a large number of BPS solutions with the same asymptotic charges. Second, some of the explicit solutions we find describe black rings in 10 dimensions. By varying the black-ring dipole charges we generate a large number of circular black-ring solutions with the same charges. The most general black-ring solution with a given set of asymptotic charges is obtained by specifying seven arbitrary functions with a few global constraints. Hence, these solutions copiously violate black-hole uniqueness. Third, there are quite a few D-brane probe calculations that indicate the possibility of creating geometries with closed time-like curves. ${ }^{2}$ Since our solutions should capture all BPS three-charge configurations, one can imagine trying to write down a series of BPS solutions that interpolate between a solution that has no closed time-like curves and one that has them, to try to violate chronology protection. It would be very interesting to see if this can be done, and, if not, to discover the obstructions.

In section 2, we find the equations that give all supergravity solutions that have the same supersymmetries and charges as the three-charge black hole, and then in section 3 , we give a systematic way to construct generic three-charge solutions by solving several inhomogeneous linear differential equations. Section 4 contains explicit solutions that describe supertubes

\footnotetext{
${ }^{2}$ One could do this, for example, by charging up a three-charge supertube with twodipole charges $[1,2]$. Other interesting recent work on closed time-like curves in rotating three-charge backgrounds has appeared in [14].
} 
and black rings with three charges, three dipole charges, and $U(1) \times U(1)$ symmetry, as well as solutions containing both a BMPV black hole [4] and a black ring. These solutions generalize the three equal-charge BPS blackring solutions constructed in [10] and reduce in the near ring regime to the solutions in [1]. Finally, in section 5, we offer some concluding thoughts and describe a number of interesting problems that might be addressed using the results of this paper. Our supergravity conventions are given in Appendix $\mathrm{A}$, and the BPS brane probe arguments used to constrain the metric and the Maxwell fields are given in Appendix B.

Note added: A day after this paper appeared on the arxiv, two more papers appeared that overlap with our section 4. In [9], the solution describing a circular BPS black ring with three charges and three-dipole charge was found and analyzed. In [11], solutions describing concentric BPS black rings with arbitrary charges and dipole charges in a BMPV black-hole background were found, generalizing earlier equal-charge multi-BPS-black-ring solutions [12].

\section{The equations}

In order to find the most general solution, it is best to work in a duality frame in which the symmetry between the three charges and the three dipole charges is manifest. We will, therefore, work in M-theory with three sets of M2-brane charges, which we take to be parallel to the 123, 145, and 167 planes, respectively. ${ }^{3}$ This is easily dualized to the D1-D5-p system: One first compactifies on the seven-direction and takes the T-dual along the fourand five-directions. This produces a D0-D4-F1 system in IIA supergravity, with the F1 string lying in the 16 plane. Finally, taking the T-dual in the sixdirection produces the requisite D1-D5-p system. M-theory solutions can be then mapped exactly onto D1-D5-p solutions by following the Buscher rules. Note that the directions (2.8 to 2.11 ) define an $\mathbb{R}^{4}$ that is inert under these dualities and represent the spatial part of the metric in which the supertube or black hole is localized.

From both the Born-Infeld description of three-charge supertubes [2, 22] and from the physics of other three-charge geometries $[1,10,17,23]$, we can see that three-charge solutions can also have brane dipole moments. For our M2-brane configurations, these dipole charges are magnetic and correspond to M5-branes in the $12367 y, 12345 y$, and $14567 y$ directions, where $y$ denotes

\footnotetext{
${ }^{3}$ This duality frame was first used to describe the three-charge black hole in [30].
} 
a direction in the $\mathbb{R}^{4}$ transverse to all the M2-branes. Indeed, we will consider M5-branes that wrap an arbitrary closed curve, $\vec{y}(\phi)$, in this $\mathbb{R}^{4}$.

Our purpose is to find the most general solution that preserves the same supersymmetries as the three-charge black hole. We can greatly constrain the form of the metric and the Maxwell fields by using the crucial fact that any brane probe that is mutually BPS with the three charges of our solutions should feel no force. These mutually BPS probe branes are:

- M2-branes of any orientation in the $\mathbb{R}^{4}$ transverse to the tube, parameterized by 8910 11;

- M2-branes parallel to the charges of the solution, in the 23, 45, and 67 directions;

- M5-branes which have one direction in the 23 plane, one in the 45 plane, one in the 67 plane, and the other two directions in the 891011 hyperplane.

We discuss these probes and analyze the consequences of the zero-force condition in Appendix B of this paper. Here, we simply note that the most general metric and Maxwell potential Ansatz compatible with zero force on the BPS brane probes is:

$$
\begin{aligned}
\mathrm{e}^{1}= & \mathrm{e}^{-2 A_{1}-2 A_{2}-2 A_{3}}\left(d x^{1}+\vec{k} \cdot d \vec{y}\right), \\
\mathrm{e}^{2}= & \mathrm{e}^{-2 A_{1}+A_{2}+A_{3}} d x^{2}, \\
\mathrm{e}^{3}= & \mathrm{e}^{-2 A_{1}+A_{2}+A_{3}} d x^{3}, \\
\mathrm{e}^{4}= & \mathrm{e}^{A_{1}-2 A_{2}+A_{3}} d x^{4}, \\
\mathrm{e}^{5}= & \mathrm{e}^{A_{1}-2 A_{2}+A_{3}} d x^{5}, \\
\mathrm{e}^{6}= & \mathrm{e}^{A_{1}+A_{2}-2 A_{3}} d x^{6}, \\
\mathrm{e}^{7}= & \mathrm{e}^{A_{1}+A_{2}-2 A_{3}} d x^{7}, \\
\mathrm{e}^{7+i}= & \mathrm{e}^{A_{1}+A_{2}+A_{3}} d y^{i}, \\
C^{(3)}= & -\mathrm{e}^{1} \wedge \mathrm{e}^{2} \wedge \mathrm{e}^{3}-\mathrm{e}^{1} \wedge \mathrm{e}^{4} \wedge \mathrm{e}^{5}-\mathrm{e}^{1} \wedge \mathrm{e}^{6} \wedge \mathrm{e}^{7} \\
& +2\left(\vec{a}_{(1)} \cdot d \vec{y}\right) \wedge d x^{2} \wedge d x^{3}+2\left(\vec{a}_{(2)} \cdot d \vec{y}\right) \wedge d x^{4} \wedge d x^{5} \\
& +2\left(\vec{a}_{(3)} \cdot d \vec{y}\right) \wedge d x^{6} \wedge d x^{7},
\end{aligned}
$$

where $\vec{y}$ parameterizes the $\mathbb{R}^{4}$ transverse to the branes and $A_{1}, A_{2}, A_{3}$, $\vec{k}, \vec{a}_{(1)}, \vec{a}_{(2)}$, and $\vec{a}_{(3)}$ are functions of the four coordinates $y^{i}$. Note that because $e^{1}$ has some components in the $\mathbb{R}^{4}$, the product of vielbeins in $C^{(3)}$ implicitly contains components along the transverse $\mathbb{R}^{4}$. We have chosen to write $C^{(3)}$ in this form because it makes significant simplifications to the supersymmetry variations. The harmonic functions, $Z_{i}$, that are commonly 
used to write brane metrics are:

$$
Z_{i} \equiv \mathrm{e}^{6 A_{i}}
$$

but these functions are not going to be precisely harmonic here - they will have distributed sources.

Note that the functions $Z_{i}$ implicitly appear in the components of $C^{(3)}$ parallel to the branes and thus determine the electric or "fundamental" components of the Maxwell three-form sourced by the monopolar M2-brane distributions. The Maxwell one-forms, $\vec{a}_{(j)}$, on the $\mathbb{R}^{4}$ are normal fourdimensional Maxwell fields on $\mathbb{R}^{4}$, but in the 11-dimensional theory, they govern the magnetic $F^{(4)}$ sourced by the M5-brane distribution of the solution. So, with a certain abuse of terminology, we will refer to these as the magnetic or dipolar Maxwell fields, even though they are generally electric and magnetic in the four-dimensional sense.

The key to generating our solutions is that we will construct the configurations that preserve the same Killing spinors as the three-charge black hole. The space of supersymmetries is four-dimensional and is defined by the canonical projectors for the M2-branes (see Appendix A for our conventions):

$$
\left(\mathbb{1}+\Gamma^{123}\right) \epsilon=\left(\mathbb{1}+\Gamma^{145}\right) \epsilon=\left(\mathbb{1}+\Gamma^{167}\right) \epsilon=0 .
$$

Given a supersymmetry, $\epsilon$, in M-theory, the vector

$$
K^{\mu} \equiv \bar{\epsilon} \Gamma^{\mu} \epsilon
$$

must always be a Killing vector [13]. Moreover, it must be non-zero and parallel to the time direction. To see the latter, observe that if $\Delta \equiv \Gamma^{1 A B}$ for $A B$ equal to 23,45 , or 67 , then $\Delta$ is hermitian, commutes with $\Gamma^{1}$, and satisfies $\Delta^{2}=\mathbb{1}$ with $\Delta \epsilon=-\epsilon$. One, therefore, has $\bar{\epsilon} \Delta=-\bar{\epsilon}$, and so

$$
K^{a}=\bar{\epsilon} \Delta \Gamma^{a} \Delta \epsilon= \pm K^{a},
$$

where $a$ is a frame index. To get the last equality, one commutes $\Delta$ through $\Gamma^{a}$ and uses $\Delta^{2}=\mathbb{1}$. One gets the negative sign if $\Delta$ anti-commutes with $\Gamma^{a}$, and so $K^{a}$ can only be non-zero if and only if it commutes with all choices of $\Delta$, which only happens for $a=1$. Moreover, $K^{1}=\epsilon^{\dagger} \epsilon>0$. The form of this Killing vector will be important later, but here we note that it can be used to normalize the Killing spinor because $K^{1}$ (in space-time indices) must be constant, and hence the four Killing spinors are

$$
\epsilon_{i}=e^{-A_{1}-A_{2}-A_{3}} \eta_{i},
$$

where the $\eta_{i}$ form a basis of constant spinors satisfying the projection conditions (2.12). 
Since $\Gamma^{1234567891011}=\mathbb{1}$, it follows that the Killing spinors must automatically satisfy:

$$
\left(\mathbb{1}-\Gamma^{891011}\right) \epsilon=0 .
$$

In other words, the "generalized holonomy" on the transverse $\mathbb{R}^{4}$ must be "half-flat," that is, it must be restricted to one of the $\mathrm{SU}(2)$ factors of $\mathrm{SO}(4) \equiv \mathrm{SU}(2) \times \mathrm{SU}(2)$. As we will see, this will lead to a collection of "self-duality" constraints on the background fields restricted to this $\mathbb{R}^{4}$.

The gravitino variation is given in equation (A4), and it is most convenient to consider every variation in terms of the flat or frame indices. There are three essentially distinct types of variations: those parallel to the spatial sections of the M2-branes, those in the transverse $\mathbb{R}^{4}$, and the variation in the time direction.

Because of our choice to split $C^{(3)}$ as in equation $(2.10)$, the $d \vec{k}$ terms cancel out of the variations parallel to the branes. These variations then give rise to two types of equations: the first has been already incorporated into our Ansatz via the zero-force conditions and relates the electric components of $C^{(3)}$ to the metric functions, $A_{j}$. The second set of equations is a selfduality condition on the "magnetic" Maxwell fields. Define the (two-form) field strengths on $\mathbb{R}^{4}$ by

$$
G_{j} \equiv d\left(a_{(j)}\right), \quad j=1,2,3,
$$

Then, the supergravity variations parallel to the spatial parts of the M2branes require that all the differences, $Z_{i} G_{i}-Z_{j} G_{j}$ be self-dual in $\mathbb{R}^{4}$. The variation along the time direction similarly collapses to the condition that $\sum_{i} Z_{i} G_{i}$ be self-dual, and so, we find that the individual field strengths must be self-dual:

$$
G_{i}=* G_{i},
$$

where $*$ is the flat Hodge dual on the transverse $\mathbb{R}^{4}$.

The last class of supersymmetry variations relates the vector field that governs the rotation, $\vec{k}$, to the Maxwell field, $G_{i}$ :

$$
d k+* d k=2\left(\mathrm{e}^{6 A_{1}} G_{1}+\mathrm{e}^{6 A_{2}} G_{2}+\mathrm{e}^{6 A_{3}} G_{3}\right) .
$$

Equations $(2.18,2.19)$ are all that one needs in order to satisfy the vanishing of the supersymmetry variations. However, this is not sufficient to solve the equations of motions. The simplest to solve are the Maxwell equations, and a number of them collapse to trivialities because of the self-duality of the $G_{j}$. The only non-trivial Maxwell equations arise when two of the $G_{j}$ s contribute to the $F \wedge F$ term, and the complete equation requires the 
functions $Z_{i}=\mathrm{e}^{6 A_{i}}$ to satisfy the amazingly simple equations:

$$
\begin{aligned}
& d * d Z_{1}=4 G_{2} \wedge G_{3}, \\
& d * d Z_{2}=4 G_{1} \wedge G_{3}, \\
& d * d Z_{3}=4 G_{1} \wedge G_{2} .
\end{aligned}
$$

Imposing these equations solves all of the Maxwell equations. Fortunately, we do not have to check the Einstein equations because integrability guarantees that they are automatically satisfied. This was shown in [13] to be true whenever the Killing vector, (2.13), is time-like, and this is why we were careful to examine the non-zero components of $K^{a}$ above and verify that it is indeed time-like.

Hence, the equations that give all solutions that preserve the same supersymmetry as the three-charge black hole are

$$
\begin{aligned}
G_{i} & =* G_{i}, \\
d * d Z_{i} & =2\left|\epsilon^{i j k}\right| G_{j} \wedge G_{k}, \\
d k+* d k & =2 G_{1} Z_{1}+2 G_{2} Z_{2}+2 G_{3} Z_{3},
\end{aligned}
$$

where $\left|\epsilon^{i j k}\right|$ is the absolute value of the totally anti-symmetric tensor.

When all the $Z$ s and $G$ s are equal, these equations reduce to the equations that yield all supersymmetric solutions of five-dimensional minimal supergravity [19]. One can also check that if one imposes $\mathrm{SO}(3)$ symmetry on the $\mathbb{R}^{4}$, but allows different charges, then the foregoing equations reduce to those in [1].

While we have taken the transverse space to be $\mathbb{R}^{4}$, one can easily generalize our result by replacing this by any "half-flat" four-manifold. ${ }^{4}$ That is, we can take the transverse vielbeins to be:

$$
\mathrm{e}^{7+i}=\mathrm{e}^{A_{1}+A_{2}+A_{3}} \overline{\mathrm{e}}^{i}, \quad j=1, \ldots, 4,
$$

where $\overline{\mathrm{e}}^{i}$ is the vielbein for any hyper-Kähler metric. Since the curvature tensor on such a space is self-dual, all spinors satisfying equation (2.16) will be holonomy singlets, so the hyper-Kähler connection terms will drop out of gravitino variations that involve such spinors. Thus, such a geometry will also lead to a solution that preserves four supersymmetries. While such a replacement makes no difference to the form of the equations, nontrivial hyper-Kähler manifolds have harmonic forms that can provide new

\footnotetext{
${ }^{4}$ The main reason for using a flat base space is the fact that we are seeking solutions that preserve the same supersymmetries as the three-charge black hole in flat space. Moreover, in some regime of parameters one expects our solutions to become three-charge supertubes, which can also be described as a D-brane configurations in flat space [2].
} 
components to the Maxwell fields that make up our solutions. One can also conjecture a further generalization where neither the field strength nor the connection are self-dual, but the combination of them that appears in the supersymmetry variations is.

\section{$3 \quad$ Finding solutions}

While the equations (2.21 to 2.23) exhibit non-linear relationships between the underlying fields, the actual system that one has to solve is actually linear at each step, provided that one solves it in the order presented in equations (2.21 to 2.23). As with all the linear systems that underlie brane solutions, there are choices about where to put the sources and how to arrange those sources. ${ }^{5}$ At each step, there will thus be the freedom to insert arbitrary source distributions, and the choices must be guided by the physics that we wish to describe. We would, however, note that if we want to describe a bound state, then it seems reasonable to assume that all of the branes must be sourced along the same profile. We will also see that near the ring, the leading metric coefficients are determined, via equation (2.22), by the components of the three-form flux. Thus, the geometry can sometimes respond to smooth out what could have been singularities at the sources.

Step 1. The first equation to solve is the homogeneous one, (2.21), which implies that the $G_{i}$ satisfy the Euclidean, vacuum Maxwell equations. Conversely, given a solution, $F$, to the Maxwell equations, one can take $G_{i}=$ $F+* F$. Therefore, the solution is determined by the choice of the source distribution and the boundary conditions at infinity. (It is also surprising that the equations that govern the $G_{i}$ are completely independent of the metric coefficients $A_{i} !$ ) For the solutions that we seek, the $G_{i}$ are sourced by the dipole M5-branes, and so the $G_{i}$ must fall off suitably fast at infinity, and the location and shape of these branes thus completely determine the solution to (2.21). Since there are three independent $G_{i}$ s, there are three independent source distributions to be chosen.

The field $G_{1}$ corresponds to the M5-brane that is parallel to the 4567 directions and occupies a curve in the $\mathbb{R}^{4}$. For a generalized black ring or supertube, we take this curve to be a simple closed curve. Such a configuration is dipolar and has no net M5-brane charge. Let $\vec{\mu}_{j}(\phi) \in \mathbb{R}^{4}$, for $0 \leq \phi \leq 2 \pi$, define the closed curves that source each of the fields, $G_{j}$ for $j=1,2,3$. The fields $G_{j}$ can be then obtained from the usual Green function

\footnotetext{
${ }^{5}$ For non-trivial hyper-Kähler transverse manifolds there are also additional choices of harmonic two-forms.
} 
by first calculating the vector potentials, $\vec{b}_{(j)}$, via the line integrals:

$$
\vec{b}_{(j)}(\vec{y}) \equiv \int_{0}^{2 \pi} \frac{\sigma_{j}(\phi) \vec{\mu}_{j}^{\prime}(\phi)}{\left|\vec{y}-\vec{\mu}_{j}(\phi)\right|^{2}} d \phi
$$

and then setting

$$
G_{j}=(1+*)\left(d\left(\vec{b}_{(j)} \cdot d \vec{y}\right)\right)
$$

Each of the functions, $\sigma_{j}(\phi)$, represents the $j$ th M5-brane charge density along the $j$ th curve. Since the M5-branes are spatially extended along these curves, one should take each of these functions to be a constant, proportional to the number of M5-branes stacked on each curve.

We should note that at this step, we are doing very much the same thing that was done for the two-charge, one-dipole charge metrics of $[8,24,25]$, where the shape of the dipole branes fully determines the Maxwell fields of these branes. The only difference here is that we can, in principle, have different shapes for the three dipole charges.

For the "round" black ring with three equal charges [10], or for the more general black rings and supertubes discussed in the next section, the three $G_{i}$ are sourced by the same circular profile lying in a plane in $\mathbb{R}^{4}$. However, we stress that, in general, the singularity profiles of the $G_{i}$ can be taken to be arbitrary curves, which by charge conservation must be closed. Therefore, the most general singularity profiles will be arbitrarily deformed rings.

At first sight, it may seem strange that a rotating brane can have an arbitrary profile and still be a stable BPS state, however, this is what emerges from our analysis. Probably the best way to picture it is that the M5branes are rotating around their curves at the speed of light and are thus rotationally stabilized. However, at the same time, a traveling wave is running around the M5-brane ring in the opposite direction, also at the speed of light, so that in the stationary frame of our metric, this traveling wave is stationary and thus gives rise to a general closed curve as a profile.

Step 2 Having found the $G_{i}$, one can use equation (2.22) to find the metric functions $Z_{i}$. Given a distribution of branes, the term on the right hand side of equation (2.22) contains both the explicit $G \wedge G$ term, and an implicit "fundamental" brane density, $\rho_{i}(\vec{x})$. In general, the brane densities, $\rho_{i}(\vec{x})$, need not be sourced in the same place as the dipole charges that source the $G_{j}$. 
Since the operator on the left hand side of equation (2.22) is the Laplacian on $\mathbb{R}^{4}$, it is trivial to invert, and one gets:

$$
Z_{i}(\vec{y})=c_{i}+\int \frac{\rho_{i}(\vec{z})+2\left|\epsilon^{i j k}\right| *\left(G_{j} \wedge G_{k}\right)(\vec{z})}{(\vec{y}-\vec{z})^{2}} d^{4} z
$$

where $c_{i}$ are arbitrary constants that can be set to 1 if one wants to obtain asymptotically flat solutions. Indeed, the boundary conditions are fixed by specifying the correct asymptotic geometry that contains net localized M2-brane charges.

It is quite remarkable that the combination of the fields sourced by two dipoles produces the same effect on the equations as an overall brane density. A very similar phenomenon happens in the Klebanov-Strassler solution [21], in which the RR and NS-NS three-forms transverse to the D3-branes produce a net D3-brane density dissolved in the fluxes. Hence, one can treat the right hand side of equation (2.22) as an effective brane density coming from branes dissolved into fluxes.

We should note that in the two-charge, one-dipole charge solution $[8,24$, 25], there was no brane density dissolved in fluxes because there was only one dipole charge, and the right hand side of (2.22) was identically zero.

Step 3. In order to find the rotation parameters, $\vec{k}$, one must again solve a linear inhomogeneous equation (2.23).

In fact, since this equation only gives the self-dual part of $d k$, one can add to $d k$ an arbitrary anti-self-dual form and still obtain solutions. Thus, one can, in principle, choose yet another source distribution, compute its Maxwell field, take the anti-self-dual combination of this field, then find the corresponding vector potential, and add that to $\vec{k}$. This will also solve equation (2.23). However, there is important physics in $\vec{k}$ because it describes the angular momentum of the solution, and inserting angular momentum without a corresponding matter density will generically lead to closed timelike curves. Conversely, if one has a matter density, then the freedom to add a homogeneous solutions of the equation for $\vec{k}$ can represent the freedom to spin this matter, and so such homogeneous solutions can be very important. Once again, though, there will be a danger of closed time-like curves if $\vec{k}$ becomes large, or singular. We will see an example of all this later.

Therefore, while adding an arbitrary anti-self-dual form to $d k$ might be possible in the mathematics, it can be limited or excluded by the physics of causality. 
If one acts on equation (2.23) with an exterior derivative, one obtains the Maxwell's equation for $\vec{k}$ :

$$
* d * d k=* 2\left[\left(d Z_{1}\right) \wedge G_{1}+\left(d Z_{2}\right) \wedge G_{2}+\left(d Z_{3}\right) \wedge G_{3}\right] \equiv J .
$$

The current $J$ is trivially conserved, and so one can find a solution to equation (3.4) by standard methods of electromagnetism. Indeed, one should note that the change of variables $t \rightarrow t+\psi(y)$ induces a gauge transformation, $\vec{k} \rightarrow \vec{k}+\vec{\nabla} \psi$. Thus, one can work in Lorentz gauge $(* d * k=0)$ and use the Green function of the Laplacian to define a vector field:

$$
\vec{\ell}(\vec{y}) \equiv \int_{\mathbb{R}^{4}} \frac{\vec{J}}{|\vec{y}-\vec{z}|^{2}} d^{4} z .
$$

This vector field is almost $\vec{k}$. Consider the two-form

$$
H \equiv(1+*) d \ell-2 Z_{1} G_{1}-2 Z_{2} G_{2}-2 Z_{3} G_{3},
$$

which, by construction, satisfies

$$
d H=d * H=0,
$$

even at the sources. Since on $\mathbb{R}^{4}$ the topology is trivial, $H$ can always be written as $d B$, and hence, the most general solution to equation (2.23) is

$$
k=\ell+\frac{B}{2}+k_{\mathrm{ASD}},
$$

where $k_{\mathrm{ASD}}$ is the undetermined anti self-dual part of $k$ :

$$
(1+*) d k_{\mathrm{ASD}}=0 .
$$

In practice, $H$ is usually going to be zero. Since it is a harmonic twoform (even at the sources), it is completely determined by the boundary conditions and the topology. Since the $Z_{i} G_{i}$ fall off rapidly at infinity, nontrivial $H$ s cannot come from non-normalizable modes, and so $H$ must be zero in $\mathbb{R}^{4}$. It is amusing to note that there is a danger for non-trivial topology: for equation (2.23) to be solvable, $\sum_{j} Z_{j} G_{j}$ must be orthogonal to all harmonic two-forms.

Hence, finding a solution is a three-step process, involving the solving of three sets of linear equations. The first step is to find the self-dual forms $G_{i}$ from the shapes of the dipole branes. The second is to use this solution and the brane densities to find the harmonic functions $Z_{i}$. The third is to use $Z_{i}$ and $G_{i}$ to find the rotation parameters, $\vec{k}$.

Of course, actually solving these three sets of equations for generic dipole profiles is quite non-trivial and involves non-trivial integrals. However, the whole process is linear and hence presents no obstructions. We can also see that near the dipole profile the solution becomes that of the three-charge flat supertube, or black tube, [1]. Since that solution has a small Ricci scalar and 
is free of singularities, one can infer that the most general solutions will be non-singular, provided that the three dipole profiles are identical. Moreover, when a certain combination of the local charge densities, $\rho_{j}(\vec{y})$, and dipole charges is exactly equal to zero, these solutions become regular, three-charge supertubes of arbitrary shape. When this combination is greater than zero, the solutions become black rings of arbitrary shape. When it is less than zero, the solutions have closed time-like curves and are not physical.

In the next section, we will use the procedure outlined in this section to construct asymptotically flat BPS black ring and supertube solutions with $U(1) \times U(1)$ invariance and arbitrary charges and dipole charges. We also construct a solution describing the black ring in the background of a rotating three-charge black hole.

\section{Examples}

Using our general linear system, it is easy to find the most general black-ring solution with three charges, three dipole charges, and $U(1) \times U(1)$ symmetry. This black ring was conjectured to exist in [2] using Mathur's picture of black holes. The near ring limit of its metric was obtained in [1], and the full ring solution with equal charges and equal dipole charges was obtained in [10]. Other interesting work on black rings has appeared in [7]. Our general ring solution can be further generalized to a solution containing both a black ring and a BMPV black hole, and so we present both results to illustrate the power of the method and to show how the obviously desirable superposition principle operates in this system. Since the non-linearities appear only in source terms for geometric quantities, we can find the exact non-linear consequences of how two BPS objects affect one another and, in particular, modify their respective horizons and entropies. A different superposition principle for $U(1) \times U(1)$ invariant geometries with three equal charges has been used in [12] to obtain superpositions of black tubes and black holes. It would be interesting to see if that construction extends to the more generic case considered here.

\subsection{Black rings with arbitrary charges and dipole charges}

If one assumes $U(1) \times U(1)$ symmetry, then it is natural to pass to two sets of polar coordinates $\left(z, \theta_{1}\right)$ and $\left(r, \theta_{2}\right)$ in which:

$$
d \vec{y} \cdot d \vec{y}=\left(d z^{2}+z^{2} d \theta_{1}^{2}\right)+\left(d r^{2}+r^{2} d \theta_{2}^{2}\right) .
$$


We will locate the ring at $r=0$ and $z=R$. The Maxwell fields, $\vec{a}_{(j)}$, in equation (2.10) must respect the $U(1)$ symmetries, and so we take:

$$
\vec{a}_{(j)} \cdot d \vec{y}=c_{j}(z, r) d \theta_{1}+d_{j}(z, r) d \theta_{2},
$$

where $c_{j}$ and $d_{j}$ are arbitrary functions. In principle, there could be $z$-components and $r$-components to the $\vec{a}_{(j)}$, leading to a field strength term proportional to $d r \wedge d z$. However, self-duality and the Bianchi identities mean that there would have to be a term exactly of the form const. $d \theta_{1} \wedge d \theta_{2}$, and this would lead to singular geometry at infinity.

While we could continue to work on these coordinates, it is better to work in a form of bipolar coordinates that makes the ring appear simpler and most particularly leads to the simplest possible expressions for the self-dual $G_{j}$. We want the $G_{j}$ to be simple because they generate the sources for the other equations in our linear system.

The best coordinate system to use is the one in [10], in which the flat $\mathbb{R}^{4}$ metric has the form:

$$
d s_{\mathbb{R}^{4}}^{2}=\frac{R^{2}}{(x-y)^{2}}\left(\frac{d y^{2}}{y^{2}-1}+\left(y^{2}-1\right) d \theta_{1}^{2}+\frac{d x^{2}}{1-x^{2}}+\left(1-x^{2}\right) d \theta_{2}^{2}\right) .
$$

and the self-dual ${ }^{6}$ field strengths are

$$
G_{i}=q_{i}\left(d x \wedge d \theta_{2}-d y \wedge d \theta_{1}\right)
$$

The actual change of variables is:

$$
\begin{aligned}
& x=-\frac{z^{2}+r^{2}-R^{2}}{\sqrt{\left((z-R)^{2}+r^{2}\right)\left((z+R)^{2}+r^{2}\right)}}, \\
& y=-\frac{z^{2}+r^{2}+R^{2}}{\sqrt{\left((z-R)^{2}+r^{2}\right)\left((z+R)^{2}+r^{2}\right)}},
\end{aligned}
$$

and if we express the $G_{i}$ in terms of the $z$ and $r$ coordinates, we find exactly the Maxwell forms sourced by a circular, uniform dipole profile in equation (3.1). The $(x, y)$ coordinates lie in the ranges $-1 \leq x \leq 1$, $-\infty<y \leq-1$, and the ring is located at $y=-\infty$.

\footnotetext{
${ }^{6}$ Our orientation is $\epsilon^{y x \theta_{1} \theta_{2}}=1$.
} 
Since the $G_{j}$ are so simple, it is quite an easy exercise to find the form of the $Z_{i}$ that satisfies equation $(2.22)$ :

$$
\begin{aligned}
& Z_{1}=1+\frac{Q_{1}}{R}(x-y)-\frac{4 q_{2} q_{3}}{R^{2}}\left(x^{2}-y^{2}\right), \\
& Z_{2}=1+\frac{Q_{2}}{R}(x-y)-\frac{4 q_{1} q_{3}}{R^{2}}\left(x^{2}-y^{2}\right), \\
& Z_{3}=1+\frac{Q_{3}}{R}(x-y)-\frac{4 q_{1} q_{2}}{R^{2}}\left(x^{2}-y^{2}\right) .
\end{aligned}
$$

The terms proportional to $q_{j} q_{k}$ are sourced by $G_{j} \wedge G_{k}$, while the terms proportional to $Q_{i}$ correspond to choosing a uniform "fundamental" charge distribution, $\rho_{i}$, in equation (3.3). Indeed, one should note that the functional dependence of these terms is:

$$
x-y=\frac{2 R^{2}}{\sqrt{\left((z-R)^{2}+r^{2}\right)\left((z+R)^{2}+r^{2}\right)}}
$$

which shows that the $Q_{i}$ terms are precisely sourced by a circular ring of radius $R$ with constant charge densities and that the $Q_{i}$ are proportional to the charge densities.

The self-dual two-form that appears on the right hand side of equation $(2.23)$ is

$$
2 \sum_{i=1}^{3} Z_{i} G_{i}=\left[A+B(x-y)+C\left(x^{2}-y^{2}\right)\right]\left(d x \wedge d \theta_{2}-d y \wedge d \theta_{1}\right),
$$

where

$$
\begin{aligned}
A & \equiv 2\left(q_{1}+q_{2}+q_{3}\right), \quad B \equiv \frac{2}{R}\left(Q_{1} q_{1}+Q_{2} q_{2}+Q_{3} q_{3}\right), \\
C & \equiv-\frac{24 q_{1} q_{2} q_{3}}{R^{2}}
\end{aligned}
$$

Hence, the components of the one-form, $k$, satisfy the equations:

$$
\begin{gathered}
\left(y^{2}-1\right) \partial_{y} k_{2}+\left(1-x^{2}\right) \partial_{x} k_{1}=0, \\
\partial_{x} k_{2}-\partial_{y} k_{1}=A+B(x-y)+C\left(x^{2}-y^{2}\right) .
\end{gathered}
$$

If we now define $p_{1}$ and $p_{2}$ by:

$$
\begin{aligned}
& k_{1}=p_{1}+(\alpha-1) A y+\frac{1}{2} B y^{2}+\frac{1}{3} C y^{3}, \\
& k_{2}=p_{2}+\alpha A x+\frac{1}{2} B x^{2}+\frac{1}{3} C x^{3},
\end{aligned}
$$

for some freely choosable parameter $\alpha$, then one has

$$
\left(1-y^{2}\right) \partial_{y} p_{2}-\left(1-x^{2}\right) \partial_{x} p_{1}=0, \quad \partial_{x} p_{2}-\partial_{y} p_{1}=0
$$


One can then differentiate and eliminate either $p_{1}$ or $p_{2}$. Indeed, eliminating $p_{2}$ yields a simple partial differential equation for $p_{1}$ :

$$
\left(1-y^{2}\right) \partial_{y}^{2} p_{1}-\partial_{x}\left(\left(1-x^{2}\right) \partial_{x} p_{1}\right)=0
$$

This is trivially separable, and one is led to the ODEs:

$$
\begin{gathered}
\left(1-x^{2}\right) \frac{d^{2}}{d x^{2}} f(x)-2 x \frac{d}{d x} f(x)+\lambda f(x)=0, \\
\left(1-y^{2}\right) \frac{d^{2}}{d y^{2}} g(y)+\lambda g(y)=0,
\end{gathered}
$$

where $\lambda$ is a separation constant. The first of these equations is the Legendre equation, and if one differentiates the second equation with respect to $y$, then one sees that $g^{\prime}(y)$ must also satisfy the Legendre equation.

The precise form of Legendre function depends upon the boundary conditions. Recall that $-1<x<1$ and $-\infty<y<-1$, and:

$$
\begin{gathered}
x=-1 \Leftrightarrow r=0, \quad z>R ; \quad x=+1 \Leftrightarrow r=0, \quad z<R \\
y=-\infty \Leftrightarrow r=0 ; \quad z=R, \quad y=-1 \Leftrightarrow z=0 .
\end{gathered}
$$

We do not want any singularities inside or outside the ring and so $f(x)$ must be regular at \pm 1 . This means that $\lambda=n(n+1)$ for some integer, $n \geq 0$, and $f(x)=P_{n}(x)$, where $P_{n}(x)$ is the corresponding Legendre polynomial. Given that $\lambda=n(n+1)$, the function $g^{\prime}(y)$ must be a combination of $P_{n}(y)$ and the second Legendre function, $Q_{n}(y)$. However, the latter has a logarithmic singularity at $y=-1$, and hence we have $g^{\prime}(y)=P_{n}(y)$.

Define $\widehat{P}_{n}(y)=\int P_{n}(y) d y$, where the arbitrary constant of integration is fixed by requiring that $\widehat{P}_{n}(y)$ satisfy the second equation of (4.18). Then the most general regular solution to (4.17) is of the form:

$$
p_{1}=\sum_{m=0}^{\infty} a_{n} P_{n}(x) \widehat{P}_{n}(y), \quad p_{2}=\sum_{m=0}^{\infty} a_{n} \widehat{P}_{n}(x) P_{n}(y),
$$

where $a_{n}$ are arbitrary constants. We can now fix all of these constants by examining the asymptotics and requiring that there be no time-like curves near the ring.

The ring is located at $r=0, z=R$, or at $y=-\infty$, and the surface of the ring can be described by $\left(x, \theta_{1}, \theta_{2}\right)$. The $(r, z)$ coordinates are singular in this region, indeed setting $z=R+\epsilon$ and $r=|\epsilon \tan \psi|$, one finds that, to zeroth order in $\epsilon, x=-\cos \psi$. Thus, one of the coordinates on the surface of the ring involves the ratio $r /(z-R)$. We will thus use the $\left(x, y, \theta_{1}, \theta_{2}\right)$ coordinates and since $-1 \leq x \leq 1$ globally, we will set $x=-\cos \psi$. 
Along the ring, the metric reduces to the three-metric:

$$
d s_{3}^{2}=\frac{V^{2} R^{2}}{(x-y)^{2}}\left[\left(y^{2}-1\right) d \theta_{1}^{2}+d \psi^{2}+\sin ^{2} \psi d \theta_{2}^{2}\right]-V^{-4}\left(k_{1} d \theta_{1}+k_{2} d \theta_{2}\right)^{2},
$$

where

$$
V \equiv\left(Z_{1} Z_{2} Z_{3}\right)^{1 / 6}
$$

The obvious danger is that if the second term dominates, then it is possible to have closed time-like curves, and this danger is most acute when $y \rightarrow-\infty$. In this limit, one has $V^{2} \sim\left(C^{2} / 9 R^{2}\right)^{1 / 6} y^{2}$, and so $p_{1}$ can, at most, be cubic in $y$ and $p_{2}$ can, at most, be quadratic in $y$. Since $\widehat{P}_{2}(y)$ is actually a cubic, this means that one must take $a_{n}=0, n \geq 3$ in equation (4.21). It turns out that one must also take $a_{2}=0$. This is because the $y^{2} d \theta_{1}^{2}$ terms are proportional to $C^{2}-\left(C+\gamma a_{2} P_{2}(x)\right)^{2}$ for some constant, $\gamma$. Near the tube, $x$ can vary from -1 to 1 , depending on the approach angle, $\psi$, and because $P_{2}(x)$ changes sign in this range, there will always be a range of $x$ values in which closed time-like curves occur unless we take $a_{2}=0$. Thus, the $y^{2} d \theta_{1}^{2}$ terms must cancel exactly.

Next, there are terms of the form $y d \theta_{1}^{2}$, and these have a coefficient proportional to $x\left((C / 3)-\left(a_{1} / 2\right)\right)$. Again, since $-1<x<1$ near the ring, there will always be a region in which this term is dominant and negative, giving rise to closed time-like curves, unless we take $a_{1} / 2=C / 3$. The constant $a_{0}$ can be absorbed into the parameter $\alpha$ introduced in equation (4.15). It is also possible to add arbitrary constants, $b_{j}$, to each of the $p_{j}$, but these can be fixed by requiring that the $k_{j}$ vanish at infinity.

There is one last subtlety in the issue of closed time-like curves. Above, we have collected the leading terms in the metric in the limit $y \rightarrow-\infty$. However, some of these leading terms vanish at $x= \pm 1$, and so we must examine sub-leading terms, and even though they are vanishing as $y \rightarrow-\infty$, they can still give rise to closed time-like curves. Indeed, one finds such problematic terms at $x=1$, and they are proportional to $-\alpha^{2}$. This means that one must also set $\alpha \equiv 0$. The term proportional to $\alpha$ is an anti-self-dual contribution to $\vec{k}$ sourced on the ring and corresponds to spinning the ring along the $s^{2}$ of the horizon. Our solution indicates that this is not possible, despite the presence of a non-trivial matter density on the ring. ${ }^{7}$

\footnotetext{
${ }^{7}$ One can also show that the flat black ring is dual to the four-charge four-dimensional BPS black hole. Hence, the fact that $\alpha$ must be zero is not surprising, since a non-zero $\alpha$ corresponds to giving this black hole angular momentum.
} 
The end result is:

$$
\begin{aligned}
& k_{1}=\left(y^{2}-1\right)\left(\frac{C}{3}(x+y)+\frac{B}{2}\right)-A(y+1), \\
& k_{2}=\left(x^{2}-1\right)\left(\frac{C}{3}(x+y)+\frac{B}{2}\right) .
\end{aligned}
$$

In terms of usual $\mathbb{R}^{4}$ coordinates, the solution for the black ring can be written as:

$$
\begin{aligned}
Z_{i}= & 1+\frac{2 R Q_{i}}{\Sigma}+\frac{8\left|\epsilon^{i j k}\right| q_{i} q_{j}\left(r^{2}+z^{2}\right)}{\Sigma^{2}}, \\
k_{1}= & \frac{4 R^{2} z^{2}}{\Sigma^{2}}\left(\frac{Q_{1} q_{1}+Q_{2} q_{2}+Q_{3} q_{3}}{R}+\frac{16 q_{1} q_{2} q_{3}\left(r^{2}+z^{2}\right)}{R^{2} \Sigma}\right) \\
& +\frac{8 R^{2}\left(q_{1}+q_{2}+q_{3}\right) z^{2}}{\Sigma\left(\Sigma+r^{2}+z^{2}+R^{2}\right)}, \\
k_{2}= & -\frac{4 R^{2} r^{2}}{\Sigma^{2}}\left(\frac{Q_{1} q_{1}+Q_{2} q_{2}+Q_{3} q_{3}}{R}+\frac{16 q_{1} q_{2} q_{3}\left(r^{2}+z^{2}\right)}{R^{2} \Sigma}\right), \\
C^{(3)}= & -\mathrm{e}^{1} \wedge \mathrm{e}^{2} \wedge \mathrm{e}^{3}-\mathrm{e}^{1} \wedge \mathrm{e}^{4} \wedge \mathrm{e}^{5}-\mathrm{e}^{1} \wedge \mathrm{e}^{6} \wedge \mathrm{e}^{7} \\
& +\left(\frac{z^{2}+r^{2}+R^{2}}{\sqrt{\left((z-R)^{2}+r^{2}\right)\left((z+R)^{2}+r^{2}\right)}} d \theta_{1}\right. \\
& \left.-\frac{z^{2}+r^{2}-R^{2}}{\sqrt{\left((z-R)^{2}+r^{2}\right)\left((z+R)^{2}+r^{2}\right)}} d \theta_{2}\right) \\
& \wedge\left(2 q_{1} d x^{2} \wedge d x^{3}+2 q_{2} d x^{4} \wedge d x^{5}+2 q_{3} d x^{6} \wedge d x^{7}\right),
\end{aligned}
$$

where $\Sigma$ is the inverse of the harmonic function sourced by the ring:

$$
\Sigma \equiv \sqrt{\left((z-R)^{2}+r^{2}\right)\left((z+R)^{2}+r^{2}\right)} .
$$

With these expressions for $k_{j}$, one can easily check that the three-metric (4.22) has the following limit as $y \rightarrow-\infty$ :

$$
d s_{3}^{2}=\left(\frac{C^{2}}{9 R^{2}}\right)^{1 / 3}\left[\left(\frac{9 R^{2}}{C^{2}}\right) M d \theta_{1}^{2}+R^{2}\left(d \psi^{2}+\sin ^{2} \psi\left(d \theta_{1}+d \theta_{2}\right)^{2}\right)\right],
$$

where

$$
\begin{aligned}
M \equiv & \left(2 q_{1} q_{2} Q_{1} Q_{2}+2 q_{1} q_{3} Q_{1} Q_{3}+2 q_{2} q_{3} Q_{2} Q_{3}-q_{1}^{2} Q_{1}^{2}-q_{2}^{2} Q_{2}^{2}-q_{3}^{2} Q_{3}^{2}\right) \\
& +\frac{1}{3} A C R^{2} .
\end{aligned}
$$

We, therefore, see that the ring does indeed has the geometry of $S^{2} \times S^{1}$ and that the horizon has a volume of $8 \pi^{2} M R^{2}$ and a cross-sectional area of 
$4 \pi\left(1 / 9\left(C^{2} R^{4}\right)\right)^{1 / 3}$. This near-ring metric reproduces exactly the metric of the flat ring found in [1] after identifying $q_{i}=d_{i}$.

The supertube solutions are those with $M=0$ and hence have zero horizon area. As $y \rightarrow-\infty$, the warp factors in front of the spatial part of the M2-branes go to a finite limit of the form $\left(q_{i} q_{j} / q_{k}^{2}\right)^{1 / 3}$, while the remaining five-dimensional part of the space time becomes $A d S_{3} \times S^{2}$, albeit with a null orbifold in the $A d S_{3}$. The full 11-metric is thus regular for $M=0$. It is also important to note that at least the Ricci scalar, and presumably all the other curvature invariants are of order $\left(q_{1} q_{2} q_{3}\right)^{-1 / 3}$, and so even relatively modest dipole charges lead to a solution whose curvatures can be kept away from the string scale. Therefore, the supergravity approximation can be trusted as a description of these backgrounds and thus, if Mathur [27] is correct, as a description of black-hole microstates.

One can also express the entropy and the angular momenta of the black ring in terms of the quantized ring charges, $\bar{N}_{i}$, and dipole charges, $n_{i}$, which are related to $Q_{i}$ and $q_{i}$ via:

$$
Q_{i}=\frac{\bar{N}_{i} l_{p}^{6}}{2 L^{4} R}, \quad q_{i}=\frac{n_{i} l_{p}^{3}}{4 L^{2}},
$$

where $L$ is the length of the two-tori. The asymptotic charges, $N_{i}$, of the solution are the sum of the charges on the black ring, $\bar{N}_{i}$, and the charges dissolved in fluxes:

$$
N_{1}=\bar{N}_{1}+n_{2} n_{3}, \quad N_{2}=\bar{N}_{2}+n_{1} n_{3}, \quad N_{3}=\bar{N}_{3}+n_{1} n_{2} .
$$

Similarly, the angular momenta have both a contribution from the ring and a contribution from the fluxes:

$$
\begin{aligned}
& J_{1}=J^{T}+\left(n_{1} n_{2} n_{3}+\sum_{i=1}^{3} \frac{n_{i} \bar{N}_{i}}{2}\right)=J^{T}+\frac{1}{2}\left(\sum_{i=1}^{3} n_{i} N_{i}-n_{1} n_{2} n_{3}\right) \\
& J_{2}=-\left(n_{1} n_{2} n_{3}+\sum_{i=1}^{3} \frac{n_{i} \bar{N}_{i}}{2}\right)=-\frac{1}{2}\left(\sum_{i=1}^{3} n_{i} N_{i}-n_{1} n_{2} n_{3}\right)
\end{aligned}
$$

where $J^{T}$ is the angular momentum carried by the ring. Even if both $J^{T}$ and $n_{i}$ are quantized, supersymmetry requires them to be related:

$$
J^{T}=\frac{R^{2} L^{4}}{l_{p}^{6}}\left(n_{1}+n_{2}+n_{3}\right) .
$$

This relation determines the radius of the ring. 
The entropy is simply

$$
S=\frac{2 \pi A}{\kappa_{11}^{2}}=\pi \sqrt{\mathcal{M}}
$$

where

$$
\begin{aligned}
\mathcal{M}= & 2 n_{1} n_{2} \bar{N}_{1} \bar{N}_{2}+2 n_{1} n_{3} \bar{N}_{1} \bar{N}_{3}+2 n_{2} n_{3} \bar{N}_{2} \bar{N}_{3}-n_{1}^{2} \bar{N}_{1}^{2}-n_{2}^{2} \bar{N}_{2}^{2} \\
& -n_{3}^{2} \bar{N}_{3}^{2}-4\left(J_{1}+J_{2}\right) n_{1} n_{2} n_{3} \\
= & 2 n_{1} n_{2} N_{1} N_{2}+2 n_{1} n_{3} N_{1} N_{3}+2 n_{2} n_{3} N_{2} N_{3}-n_{1}^{2} N_{1}^{2}-n_{2}^{2} N_{2}^{2}-n_{3}^{2} N_{3}^{2} \\
& -n_{1} n_{2} n_{3}\left[4\left(J_{1}+J_{2}\right)+2\left(n_{1} N_{1}+n_{2} N_{2}+n_{3} N_{3}\right)-3 n_{1} n_{2} n_{3}\right] .
\end{aligned}
$$

We can see that given the three asymptotic charges, $N_{i}$, and the two angular momenta, there is still a two parameter family of circular black rings with those charges. (There are seven parameters, $\left(N_{i}, n_{j}, R\right)$ and five asymptotic charges.) Hence, the circular black rings alone copiously violate black-hole uniqueness.

\subsection{A black ring in a rotating black-hole background}

In order to describe a black ring in the background of a rotating three-charge black hole, one must add to the harmonic functions, $Z_{i}$, a component coming from a black hole at the origin. If the black hole has charges $Y_{1}, Y_{2}$, and $Y_{3}$, the harmonic functions become:

$$
\begin{aligned}
& Z_{1}=1-\frac{Y_{1}}{R^{2}} \frac{x-y}{x+y}+\frac{Q_{1}}{R}(x-y)-\frac{4 q_{2} q_{3}}{R^{2}}\left(x^{2}-y^{2}\right), \\
& Z_{2}=1-\frac{Y_{2}}{R^{2}} \frac{x-y}{x+y}+\frac{Q_{2}}{R}(x-y)-\frac{4 q_{1} q_{3}}{R^{2}}\left(x^{2}-y^{2}\right), \\
& Z_{3}=1-\frac{Y_{3}}{R^{2}} \frac{x-y}{x+y}+\frac{Q_{3}}{R}(x-y)-\frac{4 q_{1} q_{2}}{R^{2}}\left(x^{2}-y^{2}\right),
\end{aligned}
$$

and the equations satisfied by $k$ get an extra term from the new source:

$$
\begin{gathered}
\left(y^{2}-1\right) \partial_{y} k_{2}+\left(1-x^{2}\right) \partial_{x} k_{1}=0 \\
\partial_{x} k_{2}-\partial_{y} k_{1}=A+B(x-y)+C\left(x^{2}-y^{2}\right)+D \frac{x-y}{x+y}
\end{gathered}
$$

where

$$
D \equiv-\frac{2}{R^{2}}\left(Y_{1} q_{1}+Y_{2} q_{2}+Y_{3} q_{3}\right)
$$


The solution is:

$$
\begin{aligned}
& k_{1}=\left(y^{2}-1\right)\left(\frac{C}{3}(x+y)+\frac{B}{2}+\frac{D}{x+y}+\frac{K}{R^{2}(x+y)^{2}}\right)-A(y+1), \\
& k_{2}=\left(x^{2}-1\right)\left(\frac{C}{3}(x+y)+\frac{B}{2}+\frac{D}{x+y}+\frac{K}{R^{2}(x+y)^{2}}\right) .
\end{aligned}
$$

Here, we have added an extra homogeneous solution of the equation for $k$. That is, the term proportional to $K$ gives an anti-self-dual contribution to $d k$ and is thus annihilated by $(1+*) d$. Physically, this term represents the angular momentum of the BMPV black hole. In principle, we could have added this term to the pure black-ring solution as well. However, there will be closed time-like curves if one introduces angular momentum without accompanying mass density. More precisely, this new term will introduce closed time-like curves unless there is a black hole of charges $Y_{1} Y_{2} Y_{3}>K^{2}$ at the origin. The sign of $K$ is undetermined, so the black hole can spin in the same or in the opposite direction to the black ring.

The charges of the black hole are related to actual number of branes via

$$
N_{i}^{\mathrm{BH}}=\frac{Y_{i} L^{4}}{l_{p}^{6}}, \quad J^{\mathrm{BMPV}}=\frac{K L^{6}}{l_{p}^{6}} .
$$

By looking at the asymptotics of the solution, it is quite easy to find the angular momenta:

$$
\begin{aligned}
J_{1} & =J^{T}+\left[n_{1} n_{2} n_{3}+\sum_{i=1}^{3}\left(\frac{n_{i} \bar{N}_{i}}{2}+n_{i} N_{i}^{\mathrm{BH}}\right)+J^{\mathrm{BMPV}}\right] \\
& =J^{T}+\left[-\frac{n_{1} n_{2} n_{3}}{2}+\sum_{i=1}^{3}\left(\frac{n_{i} N_{i}}{2}+n_{i} N_{i}^{\mathrm{BH}}\right)+J^{\mathrm{BMPV}}\right], \\
J_{2} & =-\left[n_{1} n_{2} n_{3}+\sum_{i=1}^{3}\left(\frac{n_{i} \bar{N}_{i}}{2}+n_{i} N_{i}^{\mathrm{BH}}\right)+J^{\mathrm{BMPV}}\right] \\
& =-\left[-\frac{n_{1} n_{2} n_{3}}{2}+\sum_{i=1}^{3}\left(\frac{n_{i} N_{i}}{2}+n_{i} N_{i}^{\mathrm{BH}}\right)+J^{\mathrm{BMPV}}\right],
\end{aligned}
$$

where $J^{\mathrm{BMPV}}$ is the angular momentum of the BMPV black hole at the origin and $J^{T}$ is the angular momentum of the black ring (4.36). The other angular momentum terms come from the fluxes and include an interaction term between the black hole and the ring.

As expected, the solution asymptotes to the BMPV black-hole solution near the black hole and to the circular black-ring solution (4.30) near the ring. The entropy of the black hole is unchanged; however, the non-linear 
interaction between the black hole and the black ring (4.44) modifies the entropy of the latter:

$$
S=\frac{2 \pi A}{\kappa_{11}^{2}}=\pi \sqrt{\mathcal{M}}
$$

where

$$
\begin{aligned}
\mathcal{M}= & 2 n_{1} n_{2} \bar{N}_{1} \bar{N}_{2}+2 n_{1} n_{3} \bar{N}_{1} \bar{N}_{3}+2 n_{2} n_{3} \bar{N}_{2} \bar{N}_{3}-n_{1}^{2} \bar{N}_{1}^{2}-n_{2}^{2} \bar{N}_{2}^{2}-n_{3}^{2} \bar{N}_{3}^{2} \\
& -4 n_{1} n_{2} n_{3}\left(J_{1}+J_{2}+n_{1} N_{1}^{\mathrm{BH}}+n_{2} N_{2}^{\mathrm{BH}}+n_{3} N_{3}^{\mathrm{BH}}\right) .
\end{aligned}
$$

\subsection{Black rings and supertubes of arbitrary shapes}

Thus, for every set of asymptotic charges and angular momenta, there is a discretuum of circular black rings and supertubes with the same asymptotic charges.

Besides circular black rings, our results show that there will be a very large of number of black rings with the same asymptotic charges and no symmetry. As we have argued in section 3 , any closed curve in $\mathbb{R}^{4}$ gives a solution, which, near the curve, has the same metric as the flat black ring. To avoid closed time-like curves transverse to the tube, one needs to avoid adding terms in the kernel of the $(1+*) d$ operator that become singular at the tube. ${ }^{8}$ Hence, given the charges, dipole charges, and tube shape, there is no freedom in changing $k$. To avoid closed time-like curves along the tube, one must have a large enough charge density, so that $M$ is always non-negative.

There is another way to see that the circular black rings constructed here can be deformed to an arbitrary shape. It was shown by Horowitz and Marolf [20] that one can add a travelling wave along a four-charge, five-dimensional black string while keeping the metric smooth and supersymmetric. The flat four-charge black string is dual to our black ring in the large radius limit, and a travelling wave corresponds to a ripple on the ring profile. Since the ripple and the black ring move with the speed of light in opposite directions, the resulting configuration is a static deformation of the black ring.

One can also check that smoothly varying the charge densities along a flat tube [1] does not cause any problems to the solutions, as long as the constraint $M \geq 0$ is satisfied everywhere. This is not surprising, since the leading contribution to the metric near the tube comes from the dipole moments, and not from the M2-brane charges. Since the near ring metric

\footnotetext{
${ }^{8}$ These terms are similar to the terms proportional to $\alpha$ in our "round tube" solution.
} 
always approaches the flat tube one, we conclude that black-ring solutions where the charge densities vary along the ring are good solutions.

Hence, we expect a generic three-charge, three-dipole charge black ring to be determined by seven functions - four embedding functions and threecharge densities, satisfying the constraint $M>0$ everywhere on the ring. In addition to these seven functions, we also have three discrete parameters - the dipole charges. Thus, there are a huge number of black-rings, and as we mentioned in the introduction, it would be interesting to see if they overcount the entropy of the boundary D1 D5 p system.

To go from black rings to supertubes with three charges, three dipole charges, and an arbitrary shape, we only need to change the requirement $M>0$ to $M=0$ everywhere along the tube. This implies that a generic supertube metric is determined by six functions. Since the supertubes have no entropy and low curvature everywhere, their number might be large enough to account for all the microstates of the D1-D5-p system.

It also appears to be possible to obtain solutions in which $M=0$ in some sectors of the ring and $M>0$ in others. These solutions would describe black-hole beads on a supertube. It would be interesting to fully analyze these exotic configurations and see if one can freely change the horizon topology by simply moving charges around the ring. If those solutions are free of pathologies, they would provide an interesting laboratory for studying horizon topology change in a controlled BPS setting.

\section{Conclusions and future directions}

We have analyzed all 11-dimensional supergravity solutions that preserve the same supersymmetries as the three-charge black hole. Besides the three asymptotic charges, the interior of the solutions may contain up to three dipole moments associated to other types of branes. We also found that the general solution is completely determined by a simple linear system of equations that is equivalent to solving a set of charge distribution problems in four-dimensional electromagnetism. The most generic, regular solution involves seven arbitrary functions and is given by first specifying a closed curve, or "generic ring" of arbitrary shape in $\mathbb{R}^{4}$. The four arbitrary functions describing this ring determine the shape of the dipole branes. The remaining three arbitrary functions represent the densities of each of the three fundamental brane-charges and how they are spread around the ring.

Near the ring, the metric approaches the flat-ring metric of [1]. Hence, depending on how large the charge densities are, the solutions can be either 
black rings of arbitrary shape or three-charge supertubes of arbitrary shape. In section 4 , we have constructed several solutions with $U(1) \times U(1)$ symmetry, including a circular black ring/three-charge supertube with arbitrary charges and dipole charges, and the black ring in a rotating BMPV blackhole background. We have also argued that, since a solution is obtained by solving linear equations, any arbitrary ring profile in $\mathbb{R}^{4}$ with three arbitrary charge density functions gives a solution. Moreover, near the ring, the metric is free of pathologies, provided the charge densities are larger than a lower bound; therefore, these solutions are regular at least down to and across the horizon. These black rings can have the same charges and angular momenta as the BPS three-charge black hole, and thus copiously violate black-hole uniqueness. ${ }^{9}$

If the three-charge density functions satisfy a constraint, the solutions have zero horizon area, and thus have no entropy. They are also completely regular. Moreover, the scalar curvature in the core is bounded by the inverse of the cubic root of the product of the dipole charges. Thus, the supergravity approximation is valid, and these solutions are good string theory backgrounds. It is also possible to map at least some of these geometries to chiral null models $[10,15]$, and thus show they receive no string corrections [31].

While the equations are linear, the solutions to one set of equations feed non-linearly into the sources of subsequent linear equations, and so the fundamental charge distributions depend non-linearly upon the dipolar distributions, while the gravitational background depends non-linearly upon all the charge distributions. When more than one dipole charge is present, the Maxwell fields sourced by the dipole branes give rise to dissolved brane charges, much like in the Klebanov-Strassler solution [21]. Hence, the harmonic functions appearing in the metric receive a contribution both from the actual charge densities on the ring (which may vary along the ring) and from charges "dissolved" in the dipole fields. Similarly, the angular momentum of the solution contains both a direct contribution from the ring and a contribution from the Maxwell fields sourced by the dipole branes.

Our systematic construction also allows one to find solutions describing arbitrary superpositions of black rings, black holes, supertubes, and more exotic objects. Of course, the more complicated the shape of these objects, the more difficult it is to solve the inhomogeneous differential equations that

\footnotetext{
${ }^{9}$ While this might have been expected of black rings [7], the magnitude of the violation is definitely beyond expectation.
} 
give the solutions. On the other hand, the underlying system of equations is simple, and relatively well understood, and so there are many interesting, general calculations that might be done. For example, it would be very interesting to do some "maximum entropy" calculations to find the most probable configurations with given asymptotic charges. One might also examine what happens when tubes cross, or touch - this might describe a transition from a bound state to a set of states.

One of the most important things we may hope to do is to map all the zero-entropy, regular solutions to the microstates of the D1-D5-p system. If this is successful, then, as argued by Mathur [27], our picture of black holes would change at the most fundamental level: black holes would be understood as statistical ensembles of regular microstate geometries. Alternatively, one could try to count the regular solutions (in a similar way to the counting of two-charge supertubes $[3,5])$ and see if there are enough of them to account for the black-hole entropy. Of course, to really prove this conjecture, one must first determine whether our ring configurations represent true bound states. Geometry does seem to indicate a natural candidate for bound states, namely, the three-dipole charge supertubes with fundamental charges localized on the tube. This seems natural, not only by analogy with the two-charge system but also because many of these tubes have a consistent Born-Infeld description [2,22]. Nevertheless, it would be good to see this from the perspective of the dual CFT.

However, before doing any counting or mapping to the CFT, the fact remains that we have such a huge number of black rings - seven free functions worth of them. This may already be a very strong hint that this picture of black holes is valid. In this context, we also think it significant that the underlying equations are linear. This is because black-hole entropy can be described by combinations of branes, and to make larger black holes, one simply needs to combine more branes. This picture seems to implicitly need some form of superposition principle, and if there were non-linearities then one might get non-trivial restrictions on the phase space of one group of branes imposed by another group of branes. Counting would be a nightmare! At the very least, the linearity of the underlying system will make the statistics much easier to analyze.

If black holes are ensembles of geometries, then so are black rings; a black ring should be the statistical ensemble of nearby supertube geometries with the same charges. However, if black rings truly describe ensembles of microstate geometries, then it should be possible for a certain geometry to be part of more than one ensemble. If this is correct, then the entropy of the black rings with a fixed asymptotic charges should be larger than 
the corresponding D1-D5-p system entropy. It would be really interesting to see if this is indeed the case. ${ }^{10}$ This picture also makes the more exotic black-hole beads configurations easier to understand: they are simply ensembles of microstate geometries that are fixed in some regions and allowed to vary in others. The "beads" are where the ensemble averages are being taken, while the supertubes sections are where the microstate is fixed.

One can also use our solutions to construct all four-dimensional threecharge solutions that are mutually BPS with the four-dimensional black hole. The three-charge limit of this black hole has no entropy; hence, the entropy of the dual CFT cannot be described by the black-hole geometry. As in the D1-D5 system [24], it should be possible to find the microstate geometries that account for this entropy.

In addition to trying to establish Mathur's conjecture, there are quite a number of interesting related questions. One should try to understand the complicated entropy formula of circular black rings using the D1-D5-p system. What is the corresponding boundary description? What features of these microstates correspond to the dipole charges of the black ring? Given a black ring with an arbitrary shape, how can one find the corresponding boundary sector?

Then, there are questions related to more classical gravitational physics. Most of the solutions that we have found have angular momentum and are very close to having closed time-like curves. This is particularly evident in the supertube with two-dipole charges. Consider the near-ring metric parameter, $M$, in equation (4.31) for $q_{3}=0$ : One finds $M=-\left(Q_{1} q_{1}-\right.$ $\left.Q_{2} q_{2}\right)^{2}$. Thus, one would have closed time-like curves unless $\left(Q_{1} q_{1}-Q_{2} q_{2}\right) \equiv$ 0 , in which case the solution is a supertube. However, the Born-Infeld analysis [2] indicates that there is no force needed to bring a fundamental charge near a two-dipole supertube, but if one drops such a charge into the two-dipole supertube then the resulting solution would have closed time-like curves. It would be very interesting to construct the full geometry describing this process and to see if there is any obstruction to this apparent violation of chronology protection.

It is also very intriguing to analyze the more exotic configurations that have a non-zero horizon size only in several sectors of the ring. Such solutions would describe black-hole beads on a supertube, or pinched horizons, like a string of sausages. Our solutions indicate that one can go from a horizon of

\footnotetext{
${ }^{10}$ When all charges are equal, the entropy of each black ring is of order $N$, so at first glance, the family of black rings classified by seven free functions seems to definitely overcount the entropy.
} 
non-zero size to one of zero size by simply moving charges around the ring and might provide a controlled BPS setting for studying horizon topology change.

Another series of solutions one might try to construct would describe the dropping of a black ring into a black hole. Again the Born-Infeld analysis [2] suggests that this is possible and that the end result would be a BPS black hole with unequal angular momenta. Nevertheless, it has been argued [28] that such black holes do not exist.

We, therefore, believe that the results and ideas presented here will find a lot of interesting applications. We believe that we have found the most general ring solution with the same supersymmetries as the three-charge black hole, and thus we indeed have the "One Ring to Rule Them All." Probably the most intriguing of all the questions outlined here is whether they all represent bound states and whether the black hole can be thought of as a bound-state ensemble average of the regular supertube microstates. That is, does our One Ring really "in the darkness bind them?"

\section{Acknowledgments}

I.B. would like to thank Per Kraus for very stimulating discussions and insights. N.W. would like to thank the Aspen Center for Physics for its hospitality and for providing a very stimulating atmosphere while this work was completed. This work was supported in part by funds provided by the DOE under grants DE-FG03-84ER-40168 and DE-FG03-91ER-40662 and by the NSF under grants PHY00-99590 and PHY01-40151. ${ }^{11}$

\section{Appendix A: supergravity conventions}

The metric is "mostly plus," and we take the gamma-matrices to be

$$
\begin{aligned}
\Gamma_{1} & =-i \Sigma_{2} \otimes \gamma_{9}, \quad \Gamma_{2}=\Sigma_{1} \otimes \gamma_{9}, \quad \Gamma_{3}=\Sigma_{3} \otimes \gamma_{9}, \\
\Gamma_{j+3} & =\mathbb{1}_{2 \times 2} \otimes \gamma_{j}, \quad j=1, \ldots, 8,
\end{aligned}
$$

where the $\Sigma_{a}$ are the Pauli spin matrices, $\mathbb{1}_{2 \times 2}$ is the identity matrix, and the $\gamma_{j}$ are real, symmetric $\mathrm{SO}(8)$ gamma-matrices. As a result, the $\Gamma_{j}$ are

\footnotetext{
${ }^{11}$ Any opinions, findings, and conclusions expressed in this material are those of the authors and do not necessarily reflect the views of the Lord of the Rings, of the US Department of Energy, or of the National Science Foundation.
} 
all real, with $\Gamma_{1}$ skew-symmetric and $\Gamma_{j}$ symmetric for $j>2$. One also has:

$$
\Gamma^{1, \ldots, 11}=\mathbb{1},
$$

where $\mathbb{1}$ denotes the $32 \times 32$ identity matrix.

The gravitino variation is

$$
\delta \psi_{\mu} \equiv \nabla_{\mu} \epsilon+\frac{1}{288}\left(\Gamma_{\mu}^{\nu \rho \lambda \sigma}-8 \delta_{\mu}^{\nu} \Gamma^{\rho \lambda \sigma}\right) F_{\nu \rho \lambda \sigma} .
$$

With these conventions, sign choices, and normalizations, the equations of motion are:

$$
\begin{aligned}
R_{\mu \nu}+R g_{\mu \nu} & =\frac{1}{12} F_{\mu \rho \lambda \sigma} F_{\nu}^{\rho \lambda \sigma}, \\
\nabla_{\mu} F^{\mu \nu \rho \sigma} & =-\frac{1}{1152} \varepsilon^{\nu \rho \sigma \lambda_{1} \lambda_{2} \lambda_{3} \lambda_{4} \tau_{1} \tau_{2} \tau_{3} \tau_{4}} F_{\lambda_{1} \lambda_{2} \lambda_{3} \lambda_{4}} F_{\tau_{1} \tau_{2} \tau_{3} \tau_{4}} .
\end{aligned}
$$

The Maxwell equation may be written more compactly as:

$$
d * F+\frac{1}{2} F \wedge F=0 .
$$

\section{Appendix B: brane probe constraints}

In this appendix, we constrain the form of the general solution that preserves the same supersymmetries as the three-charge black hole by using the crucial fact that any brane probe that is mutually BPS with the black hole should feel no force. The first observation is that the bulk metrics must maintain the same isometries as the boundary. The three M2-branes are wrapped on $T^{2} \times T^{2} \times T^{2}$, and so any metric that mixes the $T^{2} \mathrm{~s}$ with other directions is excluded. Moreover, there cannot be any four-form field strengths with an odd number of legs along any $T^{2}$.

The solution has M2-brane charges in the 123, 145, and 167 planes. There are three types of probe branes that are mutually BPS with these charges. First, there are the M2-branes carrying these charges. Second, we can put an M2-brane along any two directions in the four-dimensional space transverse to the branes. The projector associated with these branes commutes with the three projectors which determine the Killing spinors of our solutions, and the resulting configuration preserves two supercharges. Third, we can put an M5-brane that has one direction in the 23 plane, one in the 45 plane, one in the 67 plane, and the other two directions in the 891011 hyperplane. To see that this configuration is BPS, one can pick without loss of generality the M5-brane coordinates to be 35789. If we reduce along seven, T-dualize along eight, S-dualize, and T-dualize twice along two and five we obtain a 
configuration with four D3-branes, in the 358, 248, 256, and 239 directions, which again preserves two supercharges.

The transverse M2-branes are BPS, regardless of which two of the four transverse direction they span. There are no Maxwell fields that couple to these branes, as they would correspond to giving the solution a fourth charge. Therefore, the determinant of the induced metric is constant. This implies that the $d x^{1} \wedge d x^{i} \wedge d x^{j}$ component of $\mathrm{e}^{1} \wedge \mathrm{e}^{i} \wedge \mathrm{e}^{j}$ is constant, and therefore, all transverse vielbeins are equal:

$$
\mathrm{e}_{i}^{i}=\frac{1}{\sqrt{\mathrm{e}_{1}^{1}}}
$$

Of course, there is nothing preventing $\mathrm{e}^{1}$ from having components along the $\mathbb{R}^{4}$. These components do not enter any brane action and will only be determined by the full supersymmetry equations. Hence, the transverse metric will have a flat base.

The fact that one can rotate one of the M5-brane directions in the three $T^{2} \mathrm{~S}$ on which the M2-branes giving the charges are wrapped implies that the two vielbeins of each $T^{2}$ are equal. Moreover, since there is no Maxwell field coupling with this brane, the zero force condition implies that the product of the vielbeins of the three-tori is one.

We now use the fact that branes along the 23, 45, or 67 directions feel no force. This implies that the product of the vielbeins $\mathrm{e}_{1}^{1} \mathrm{e}_{2}^{2} \mathrm{e}_{3}^{3}$ is equal to the Maxwell potential $C_{123}$, and similarly for the 45 and 67 vielbeins. This completely establishes the form of the metric and of the electric Maxwell potentials to be the one in (2.1 to 2.8$)$.

Determining the magnetic Maxwell fields is more involved. First, the $T^{2} \times T^{2} \times T^{2}$ isometry implies that all four-form field strengths can have either two legs on the same $T^{2}$, or no legs on the $T^{2} \mathrm{~s}$, or all four legs on two different $T^{2}$ s. Moreover, one can argue that forms with no legs on the $T^{2}$ s make an M2-brane in the transverse $\mathbb{R}^{4}$ feel a non-zero force; also, the supergravity equations of motion in the three-charge background imply that turning on a field strength with all four legs along two $T^{2} \mathrm{~s}$ induces a form with no legs on the $T^{2} \mathrm{~s}$, which the brane probe analysis excludes. Therefore, the only Maxwell fields compatible with our supersymmetries have two legs along either of the three two-tori. This determines the form of the Maxwell field Ansatz (2.10).

The above argument has only one gap, coming from the fact that a supergravity brane probe analysis cannot be used to find the force between two branes that can be dualized to the D0-D8 system. These D-branes are 
mutually BPS, and yet a naive analysis the Born-Infeld action of one of the brane in the background of the other gives a non-zero force. This is because there exists a RR interaction between these branes that is not captured by supergravity (for a more detailed analysis of this system see [6]). In our case the branes whose interaction with the probe M2-branes is not captured by supergravity would be D6-branes that lift to KK monopoles in M-theory. It would be interesting to see if allowing metric factors of the type these branes source would allow for more general solutions dual to black-hole hair.

\section{References}

[1] I. Bena, Splitting hairs of the three charge black hole, arXiv:hepth/0404073.

[2] I. Bena and P. Kraus, Three charge supertubes and black hole hair, arXiv:hep-th/0402144.

[3] D. Bak, Y. Hyakutake, S. Kim and N. Ohta, A Geometric look on the microstates of supertubes, arXiv:hep-th/0407253.

D. Bak, Y. Hyakutake and N. Ohta, Phase moduli space of supertubes, arXiv:hep-th/0404104.

[4] J.C. Breckenridge, R.C. Myers, A.W. Peet and C. Vafa, D-branes and spinning black holes, Phys. Lett. B 391 (1997), 93; arXiv:hepth/9602065.

[5] B. Cabrera-Palmer and D. Marolf, Counting supertubes, arXiv:hepth/0403025.

[6] U. Danielsson, G. Ferretti and I.R. Klebanov, Creation of fundamental strings by crossing D-branes, Phys. Rev. Lett. 79 (1997), 1984; arXiv:hep-th/9705084.

O. Bergman, M.R. Gaberdiel and G. Lifschytz, Branes, orientifolds and the creation of elementary strings, Nucl. Phys. B 509 (1998), 194; arXiv:hep-th/9705130.

M. Billo, P. Di Vecchia, M. Frau, A. Lerda, I. Pesando, R. Russo and S. Sciuto, Microscopic string analysis of the D0-D8 brane system and dual $R-R$ states, Nucl. Phys. B 526 (1998), 199; arXiv:hep-th/9802088.

[7] R. Emparan and H.S. Reall, A rotating black ring in five dimensions, Phys. Rev. Lett. 88 (2002), 101101; arXiv:hep-th/0110260.

H. Elvang and R. Emparan, Black rings, supertubes, and a stringy resolution of black hole non-uniqueness, JHEP 0311 (2003), 035; arXiv:hepth/0310008.

H. Elvang, A charged rotating black ring, Phys. Rev. D 68 (2003), 124016; arXiv:hep-th/0305247. 
R. Emparan, Rotating circular strings, and infinite non-uniqueness of black rings, arXiv:hep-th/0402149.

[8] R. Emparan, D. Mateos and P.K. Townsend, Supergravity supertubes, JHEP 0107 (2001), 011; arXiv:hep-th/0106012.

[9] H. Elvang, R. Emparan, D. Mateos and H.S. Reall, Supersymmetric black rings and three-charge supertubes, arXiv:hep-th/ 0408120.

[10] H. Elvang, R. Emparan, D. Mateos and H.S. Reall, A supersymmetric black ring, arXiv:hep-th/0407065.

[11] J.P. Gauntlett and J.B. Gutowski, General concentric black rings, arXiv:hep-th/0408122.

[12] J.P. Gauntlett and J.B. Gutowski, Concentric black rings, arXiv:hepth/0408010.

[13] J.P. Gauntlett and S. Pakis, The geometry of $D=11$ Killing spinors. (T), JHEP 0304 (2003), 039; arXiv:hep-th/0212008.

[14] E.G. Gimon and P. Horava, Over-rotating black holes, Goedel holography and the hypertube, arXiv:hep-th/0405019.

N. Drukker, Supertube domain-walls and elimination of closed time-like curves in string theory, arXiv:hep-th/0404239.

[15] J.B. Gutowski, D. Martelli and H.S. Reall, All supersymmetric solutions of minimal supergravity in six dimensions, Class. Quant. Grav. 20 (2003), 5049; arXiv:hep-th/0306235.

[16] J.B. Gutowski and H.S. Reall, General supersymmetric AdS(5) black holes, JHEP 0404 (2004), 048; arXiv:hep-th/0401129.

[17] S. Giusto, S.D. Mathur and A. Saxena, 3-charge geometries and their CFT duals, arXiv:hep-th/0406103.

S. Giusto, S.D. Mathur and A. Saxena, Dual geometries for a set of 3-charge microstates, arXiv:hep-th/0405017.

[18] C.N. Gowdigere, D. Nemeschansky and N.P. Warner, Supersymmetric solutions with fluxes from algebraic Killing spinors, arXiv:hepth/0306097.

K. Pilch and N.P. Warner, Generalizing the $N=2$ supersymmetric $R G$ flow solution of IIB supergravity, Nucl. Phys. B 675 (2003), 99; arXiv:hep-th/0306098.

K. Pilch and N.P. Warner, $N=1$ supersymmetric solutions of IIB supergravity from Killing spinors, arXiv:hep-th/0403005.

D. Nemeschansky and N.P. Warner, A family of M-theory flows with four supersymmetries, arXiv:hep-th/0403006.

I. Bena and N.P. Warner, A harmonic family of dielectric flow solutions with maximal supersymmetry, arXiv:hep-th/0406145. 
[19] J.P. Gauntlett, J.B. Gutowski, C.M. Hull, S. Pakis and H.S. Reall, All supersymmetric solutions of minimal supergravity in five dimensions, Class. Quant. Grav. 20 (2003), 4587; arXiv:hep-th/0209114.

[20] G.T. Horowitz and D. Marolf, Counting states of black strings with traveling waves. I + II, Phys. Rev. D 55 (1997), 835; arXiv:hep-th/ 9605224 .

G.T. Horowitz and D. Marolf, Counting states of black strings with traveling waves. I + II, Phys. Rev. D 55 (1997), 846; arXiv:hep-th/ 9606113.

[21] I.R. Klebanov and M.J. Strassler, Supergravity and a confining gauge theory: Duality cascades and $\chi S B$-resolution of naked singularities, JHEP 0008 (2000), 052; arXiv:hep-th/0007191.

I.R. Klebanov and A.A. Tseytlin, Gravity duals of supersymmetric $S U(N) \times S U(N+M)$ gauge theories, Nucl. Phys. B 578 (2000), 123; arXiv:hep-th/0002159.

[22] M. Kruczenski, R.C. Myers, A.W. Peet and D.J. Winters, Aspects of supertubes, JHEP 0205 (2002), 017; arXiv:hep-th/0204103.

[23] O. Lunin, Adding momentum to D1-D5 system, arXiv:hep-th/0404006.

[24] O. Lunin and S.D. Mathur, Metric of the multiply wound rotating string, Nucl. Phys. B 610 (2001), 49; arXiv:hep-th/0105136.

O. Lunin and S.D. Mathur, AdS/CFT duality and the black hole information paradox, Nucl. Phys. B 623 (2002), 342; arXiv:hep-th/0109154 O. Lunin and S.D. Mathur, Statistical interpretation of Bekenstein entropy for systems with a stretched horizon, Phys. Rev. Lett. 88 (2002), 211303; arXiv:hep-th/0202072.

O. Lunin, S.D. Mathur and A. Saxena, What is the gravity dual of a chiral primary?, Nucl. Phys. B 655 (2003), 185; arXiv:hep-th/0211292.

[25] O. Lunin, J. Maldacena and L. Maoz, Gravity solutions for the D1-D5 system with angular momentum, arXiv:hep-th/0212210.

[26] D. Mateos and P.K. Townsend, Supertubes, Phys. Rev. Lett. 87 (2001), 011602; arXiv:hep-th/0103030.

D. Mateos, S. Ng and P.K. Townsend, Tachyons, supertubes and brane/anti-brane systems, JHEP 0203 (2002), 016; arXiv:hepth/0112054.

[27] S.D. Mathur, A. Saxena and Y.K. Srivastava, Constructing 'hair' for the three charge hole, arXiv:hep-th/0311092.

S.D. Mathur, Where are the states of a black hole?, arXiv:hepth/0401115.

[28] H.S. Reall, Higher dimensional black holes and supersymmetry, Phys. Rev. D 68 (2003), 024024; arXiv:hep-th/0211290. 
[29] A. Strominger and C. Vafa, Microscopic origin of the BekensteinHawking entropy, Phys. Lett. B 379 (1996), 99; arXiv:hep-th/9601029.

[30] A.A. Tseytlin, Harmonic superpositions of M-branes, Nucl. Phys. B 475 (1996), 149; arXiv:hep-th/9604035.

[31] A.A. Tseytlin, Generalised chiral null models and rotating string backgrounds, Phys. Lett. B 381 (1996), 73; arXiv:hep-th/9603099. 
